overtreat rather than undertreat to prevent lifelong complications due to coronary aneurysms.

The definition of recurrent KD would essentially mean a recurrence after documented remission of the first episode of KD (clinically, echocardiography and laboratory). It goes without saying that this period would be at least for about 4 to 6 weeks.

This is a position paper on KD providing diagnostic and therapeutic guidelines for practising pediatricians across the country. We did not intend to highlight or analyze Indian data. Moreover, data on KD in India is predominantly emerging from few centres and not representative of the scenario in the whole country.

BHASKAR SHENOY Department of Pediatrics, Manipal Hospitals, Bangalore, Karnataka, India. bshenoy@gmail.com

\section{Inclusion of Multisystem Inflammatory Syndrome in Children and Adolescents Temporally Related to COVID -19 in the Differential Diagnosis of Kawasaki Disease}

It was an interesting and informative read to go through the IAP position paper on Kawasaki disease (KD) published recently [1]. It was indeed necessary to have a nation-wide consensus, which is suitable for India where the distribution of health resources is unequal and constrained. KD has become one of the leading causes of acquired heart disease in many developed countries of the world [1]. With almost a year of coronavirus disease 19 (COVID-19) pandemic, a new hyper-inflammatory syndrome affecting children has been observed, and variously labeled as Multisystem inflammatory syndrome in children (MIS-C) associated with COVID-19 or Pediatric inflammatory multisystem syndrome-temporally associated with SARS-CoV-2 (PIMS-TS) [2,3]. This condition has clinical features which overlap with other inflammatory diseases in childhood like Kawasaki disease (KD) and toxic shock syndrome (TSS) [2].

The position paper [1] mentions various differential diagnosis of KD, but does not mention PIMS-TS or MIS-C. It is important that this entity should be considered in the differential diagnosis of $\mathrm{KD}$, as the clinical presentation is very much overlapping, though the underlying mechanism for hyperinflammation is different. In KD inflammation of the coronary arteries is due to IL-1, the myocardial dysfunction and higher severity of the 2019-nCoV infection is predominantly driven by IL-6 and IL-10 in MIS-C [4]. Three different phenotypes of hyperinflammation in children has been speculated as classic KD, PIMS-TS and macrophage activation syndrome [4]. Though IVIG and steroids are the mainstay of therapy in these conditions and aspirin also important in $\mathrm{KD}$, the prognosis and long term follow up are different. Hence the authors would like to suggest that this evolving inflammatory disease should be included in the position paper as one of the differentials for KD.

Tushar Jagzape* ANd ANIL Kumar Goel Department of Pediatrics, AIIMS, Raipur, Chattisgarh, India. *tusharjagzape@aiimsraipur.edu.in

\section{REFERENCES}

1. Shenoy B, Singh S, Ahmed MZ, Pal P, Balan S, Vishwanthan V. Indian Academy of Pediatrics Position Paper on Kawasaki Disease. Indian Pediatr. 2020;57: 1040-48.

2. Royal College of Pediatrics and Child Health. Guidance-Pediatric multisystem inflammatory syndrome temporally associated with COVID-19, 2020. Accessed December 02, 2020. Available from: https://www.rcpch.ac.uk/resources/ guidance-pediatric-multi system-inflammatory-syndrometemporally-associated covid-19

3. Bhat CS, Gupta L, Balasubramanians, Singh S, Ramanan AV. Hyperinflammatory syndrome in children associate with COVID-19: Need for awareness. Indian Pediatr. 2020;57:92935 .

4. Shulman ST. Pediatric coronavirus disease-2019-associated multisystem inflammatory syndrome. J Pediatric Infect Dis. 2020;9:285-6.

\section{AUTHORS'REPLY}

We thank the authors for raising this pertinent point. We are in total agreement that MISC (PIMS) is one of the differential diagnoses of Kawasaki disease. The draft of this article; however, was prepared prior to the onset of COVID-19 pandemic, and thus it was missed out in the list of differential diagnosis of KD in the consensus statement. The committee has modified the differential diagnosis as follows:

\section{Differential Diagnosis of Kawasaki Disease}

1. Infections - Bacterial (streptococcal, leptospirosis, rickettsia), Viral (measles, adenovirus, Epstein Barr virus).

2. Toxin related-Staphylococcal scalded skin syndrome, toxic epidermal necrolysis

3. Inflammatory-Systemic juvenile idiopathic arthritis

4. Drug hypersensitivity - Steven-Johnson syndrome, drug reaction with eosinophilia and systemic symptoms (DRESS), mercury hypersensitivity.

5. Multisystem inflammatory disease of childhood temporally related to COVID-19 (MISC-C): A condition recognized and described during the COVID-19 pandemic. This occurs largely in children, usually above 5 years of age, as a short term illness with high grade fever and often with shock with multisystem inflammation. One of the phenotypes can 


\section{CORRESPONDENCE}

resemble incomplete Kawasaki disease. This is considered to be secondary to immune dysregulation triggered after or in the later phase of the infection by severe acute respiratory syndrome coronavirus (SARS-CoV-2).
BHASKar SHENOY

Department of Pediatrics, Manipal Hospitals, Bangalore, Karnataka, India.

bshenoy@gmail.com

\section{Prehospital Management of Children with Dengue Fever Admitted in a Tertiary-Care Center}

Initial management of most children with dengue is done by local practitioners initially, and they are subsequently referred in the event of worsening of clinical condition. In the present study, we reviewed the initial management of dengue prior to being referred to our hospital, with special reference to the timing of the laboratory investigations, fluid management and use of platelet concentrates

A chart review of children referred as dengue to our hospital between September and November, 2019 was done after getting approval from the institutional ethics committee of our hospital. The data on pre-hospital management of these children recorded included: whether importance was given to packed cell volume (PCV) or platelet count; timing of dengue NS1 antigen testing, dengue $\operatorname{IgM}$ and $\operatorname{IgG}$ testing (card versus ELISA method); and treatment (fluid management, use of NSAIDs, antibiotics, and platelet transfusion). PCV is normally done at the time of presentation and repeated when clinically indicated (appearance of warning signs, progression to shock). When $\mathrm{PCV}$ is used for fluid titration, it is usually done once in 4 hours.

Among the 643 patients hospitalized for dengue during the study period, $129(20 \%)$ came by self-referral for fever because of their awareness regarding dengue. Most of the children who were brought by parents had dengue fever with no warning symptoms. $514(80 \%)$ were referred by physicians, of which a large proportion were not managed as per protocol [1]. Of the 514,385 children had dengue fever without warning symptoms, 103 children had dengue fever with warning symptoms, and 26 children had severe dengue.

Among the $80 \%$ of children who were referred from outside, in $20 \%$ of patients, PCV values were not given importance as fluids were not titrated based on PCV. On the other hand, in 30\% of patients, platelet counts were monitored thrice-a-day. Intravenous fluids were not given as per guidelines in $10 \%$ of patients, and they had received large volume of hypotonic fluids leading to signs of fluid overload, which was managed with fluid restriction and diuretics.
The timing of investigating NS1 antigen and $\operatorname{IgM}$ or IgG were not as per WHO guidelines [1], and card test was done in about $15 \%$ of patients. Despite there being no indication for platelet transfusion, $3 \%$ of patients had received platelet concentrates. Though the diagnosis of dengue was made, antibiotics were given in view of high spiking fever in $2 \%$ children. Mefenamic acid induced gastritis was seen in around $4 \%$ children. These children had normal PCV, no physical warning signs, and hence the vomiting was not considered as warning symptom and was attributed to mefenemic acid.

The children referred without warning symptoms needed just a day of observation while the ones with warning symptoms and severe dengue required four days of hospitalization. All the children in the study group improved and there was no mortality.

In a survey done in Singapore [2], where they tested the knowledge on diagnostic methods and clinical management of dengue using a questionnaire, there were significant issues in the understanding and diagnosis of dengue, particularly on the importance of using a diagnostic kit. There were also significant increase in awareness and practices of the best practices of dengue clinical management (choice of fluids and use of platelet concentrates) [2].

It is important to make sure that the protocols are uniformly followed by practitioners to ensure timely referral which in turn improves the outcome and reduces mortality. The present study underscores the gaps in knowledge about dengue management among practitioners, and we plan to conduct training activities for the same.

\section{Janani SankaR* AND Nithya Saravanan Kanchi Kamakoti Childs Trust Hospital Chennai, India. \\ *janani.sankar@yahoo.com}

\section{REFERENCES}

1. World Health Organization. National guidelines for clinical management of dengue fever. WHO Country Office for India, 2015. Available from https://apps.who.int/iris/handle/10665/ 208893

2. Ho TS, Huang MC, Wang SM, Hsu HC, Liu CC. Knowledge, attitude, and practice of dengue disease among healthcare professionals in southern Taiwan. J Formos Med Assoc. $2013 ; 112: 18-23$. 